\title{
Preparation and Physical Properties of Chitosan Benzoic Acid Derivatives Using a Phosphoryl Mixed Anhydride System
}

\author{
Duckhee Lee ${ }^{1}$, Zhe Shan Quan ${ }^{2}$, Chichong Lu ${ }^{1}$, Jin Ah Jeong ${ }^{1}$, Changhyun Song ${ }^{1}$, \\ Mi-Sun Song ${ }^{1}$ and Kyu Yun Chai ${ }^{1, *}$
}

1 The Division of Bio-Nanochemistry, The College of Natural Sciences, The Wonkwang University, Iksan City, Chonbuk, 570-749, Korea; E-Mails: d1202@wonkwang.ac.kr (D.L.); chichonglu@hotmail.com (C.L.); mi_in_black@nate.com (J.A.J.); schh1004@cyworld.com (C.S.); misun4y@nate.com (M.-S.S.)

2 College of Pharmacy, Yanbian University, Yanji 133000, China;

E-Mail: quanzheshan@hanmail.net

* Author to whom correspondence should be addressed; E-Mail: geuyoon@wonkwang.ac.kr; Tel.: +82-63-850-6230; Fax: +82-63-841-4893.

Received: 1 January 2012; in revised form: 15 February 2012 / Accepted: 16 February 2012 / Published: 22 February 2012

\begin{abstract}
Direct benzoylation of the two hydroxyl groups on chitosan was achieved using a phosphoryl mixed anhydride system, derived from trifluoroacetic anhydride (TFAA), benzoic acids (BAs), and phosphoric acid (PA). The reaction is operated as a one pot process under mild conditions that does not require neither an inert atmosphere nor dry solvents. The structures of the synthesized compounds were confirmed by NMR and IR spectroscopy. Solubility tests on the products revealed that they were soluble in organic solvents such as $N, N$-dimethylformamide (DMF), dimethylsulfoxide (DMSO), and acetone. In the meantime, a morphological study by scanning electron microscopy (SEM) evidently indicated that the chitosan benzoates underwent significant structural changes after the benzoylation.
\end{abstract}

Keywords: acylation; chitosan benzoate; benzoic acid; trifluoroacetic anhydride; phosphoric acid 


\section{Introduction}

Chitin, the second abundant natural polymer in Nature, and chitosan, a partially deacetylated form of chitin, have recently received much of attention owing to their applicability in wide range of fields such as pharmaceuticals, cosmetics, agriculture, foods, and material sciences [1-5]. Their film and fiber-forming ability as well as biodegradability, biocompatibility, and abundance in Nature make them attractive materials in such application fields. However, although they have been excellent candidates for practical uses and commercialization, their uses in daily life or industry have been greatly limited due mainly to their low solubility in common solvents and poor processability. To impart better biological activity or improved physical properties like solubility in organic solvents and processibility on the polymer molecules derivatization is often performed. Modification by $O$-acylation in chitin and chitosan can be easily envisaged for such purposes. In the chitin case several methods using acid anhydride [6,7], mixed anhydride [8,9], acyl chlorides [10,11], benzoyl chloride and methanesulfonic acid [12], and p-toluenesulfonyl chloride, lithium chloride, and carboxylic acid [13] have been reported. Among them, the mixed anhydride method using trifluoroacetic anhydride, phosphoric acid, and carboxylic acid is most notable from the perspective of reaction yield, mild conditions, cheap price and recyclability of the reagents used [14-16]. This method has been already adopted by us for preparation of aliphatic and aromatic esters of chitin [17,18].

Meanwhile, in the case of chitosan, the situation is a little more complicated because there are two types of functional groups in the molecule, two hydroxyls at the 3,6-carbon position and an amino group at the 2-carbon position which is considered to be necessary for biological activities such as antibacterial or antiviral activity. Only a few acylations on chitosan are reported in the literature. Okamoto et al. used benzoyl chloride together with 4-dimethylaminopyridine functioning as a catalyst, to obtain tribenzoyl chitosan [19,20]. They also showed that $N$-(2-carboxy)benzoyl chitosan and $N$-phthaloyl chitosan derivatives could be synthesized using phthalic anhydride [21]. The acyl chloride (benzoyl) method was also employed by Feng etc. [22], Vasnev etc. [23] Zhu etc. [24], and Jiangtao [25]. The method is carried out either with acyl chloride alone or together with strong acids such as methanesulfonic acid playing role as a catalyst and simultaneously a protecting group for the free amino group.

The carboxylic acid anhydride method was often used for $N$-acylation of chitosan [26-30]. And mixed anhydride method using trifluoroacetic anhydride and carboxylic acid was also successfully applied to the synthesis of acylated chitins by Yang [8] and acylated (aliphatic) chitosans by us, respectively [31]. This method can be carried out with or without phosphoric acid. The phosphoric acid-promoted method has several advantages such as a shorter reaction sequence (one step), gentle reaction conditions, low cost of the raw materials used, simplicity of operation in preparative process, and scalability, compared to other methods mentioned above. Besides, selective esterification on the hydroxyl group attached to the primary carbon atoms of 6-positions in the glucosamine unit of chitosan polymer can be directly achieved [31].

To the best of our knowledge, nevertheless, so far, there has been no report on studies using such a reaction system for preparing aromatic carboxylic acid derivatives of chitosan. In continuation of our research on acylated chitosans, we wish to report a new synthetic method using the above reaction system for preparing chitosan benzoyl esters and a study of the physical properties of the products. 


\section{Results and Discussion}

The benzoylation of chitosan (CTS) were carried out using the mixed anhydride derived from trifluoroacetic anhydride (TFFA), benzoic acids (BAs, benzoic acid and $p$-methoxybenzoic acid), and phosphoric acid (PA). This synthetic method was chosen because it was expected to require lower molar ratios of BAs and TFFA to chitosan than the reaction using mixed anhydride derived from TFFA and BAs (Scheme 1). Both of the reactions with benzoic acid and $p$-methoxybenzoic acid proceeded smoothly in the absence of solvent to give the chitosan benzoates in good yields, although they were more sluggish than the reactions with aliphatic carboxylic acids [31]. The yields of the reaction are shown below along with the reaction conditions (Table 1).

Scheme 1. The benzoylation of chitosan using TFFA, benzoic acid, and phosphoric acid.

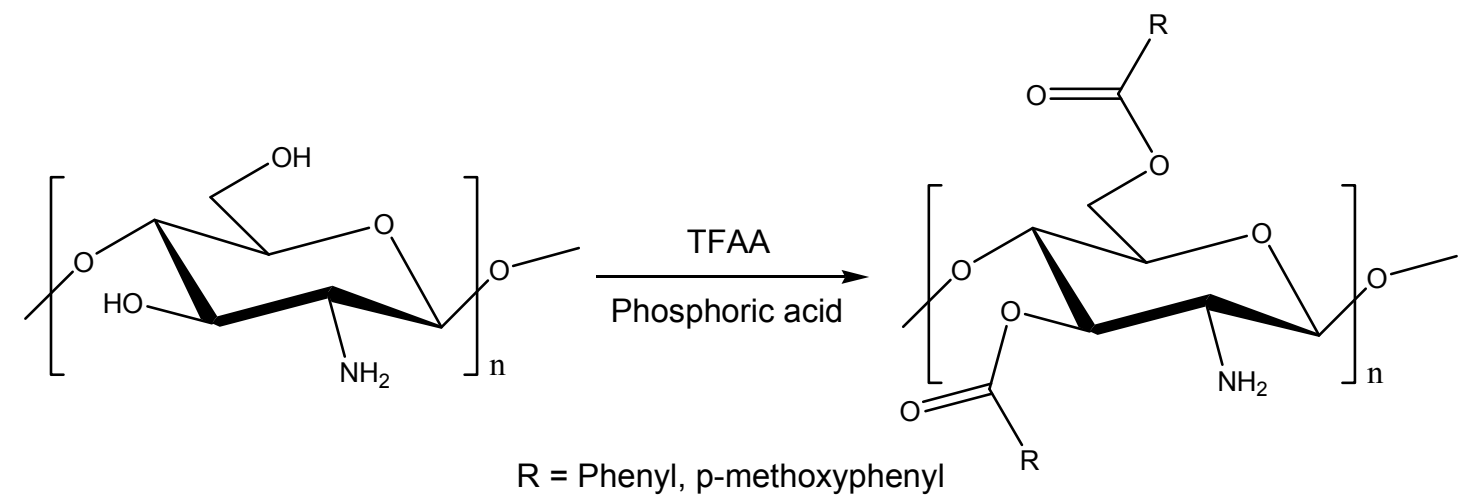

Table 1. The yields of the chitosan benzoylation using TFFA, BAs, and PA.

\begin{tabular}{cccccc}
\hline Reactants & Chitosan $(\mathrm{g})$ & TFAA $(\mathrm{g})$ & Benzoic acid $(\mathrm{g})$ & $\mathbf{H}_{\mathbf{3}} \mathbf{P O}_{\mathbf{4}}(\mathrm{g})$ & Product/Yield \\
\hline * Molar ratio & 1 & 8 & 2 & 2 & \\
Input & $1 \mathrm{~g}$ & 6.56 & 1.44 & 0.343 & ${ }^{\mathrm{a}} \mathrm{CTS}-\mathrm{b} / 1.8 \mathrm{~g}$ \\
Input & $1 \mathrm{~g}$ & 6.56 & $* * 1.80$ & 0.343 & ${ }^{\mathrm{b}} \mathrm{CTS}-\mathrm{m} / 1.5 \mathrm{~g}$ \\
\hline
\end{tabular}

* based on the glucosamine unit, ${ }^{* *} p$-methoxybenzoic acid. ${ }^{a}$ chitosan $p$-methoxybenzoate (CTS-m);

${ }^{\mathrm{b}}$ chitosan benzoate (CTS-b).

Formation of a ester bond between BAs and chitosan in the products was confirmed using infrared and NMR spectroscopy. As shown in Figure 1, a new strong and broad peak around $1,735 \mathrm{~cm}^{-1}$ corresponding to the stretching absorption of the ester carbonyl, and another strong peak around $1,210 \mathrm{~cm}^{-1}$ corresponding to the stretching absorption of the ester $\mathrm{C}-\mathrm{O}$ single bond were found in their FT-IR spectra, respectively. Both of the peaks are absent in the parent chitosan. Additional evidences for the structure of the products were obtained from the ${ }^{1} \mathrm{H}-\mathrm{NMR}$ spectra of the products shown in Figure 1, which was taken in DMSO- $d 6$ solvent with small amount of deuterated water. Characteristic signals for the protons attached to the aromatic carbon atoms and the anomeric carbon were observed between $\delta 7.5$ and $\delta 7.9$, and at $\delta 4.5$, respectively. The degree of substitution was determined to be roughly 0.89 (benzoate) and 1.0 (methoxy benzoate) respectively by integral of the peaks. 
Figure 1. The IR and NMR spectra of the benzoyl chitosans and the parent chitosan.

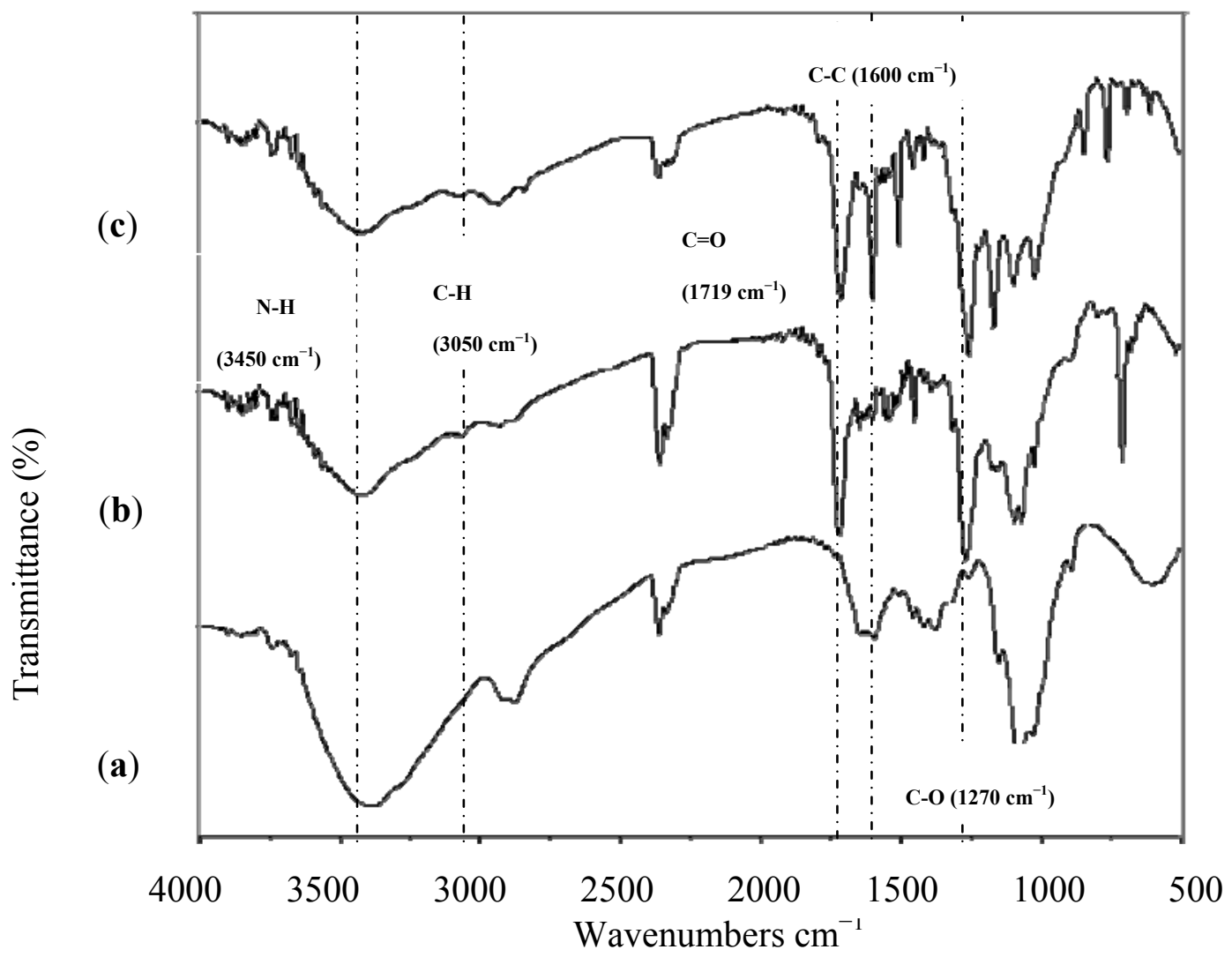

(a) CTS, (b) CTS-b, (c) CTS-m

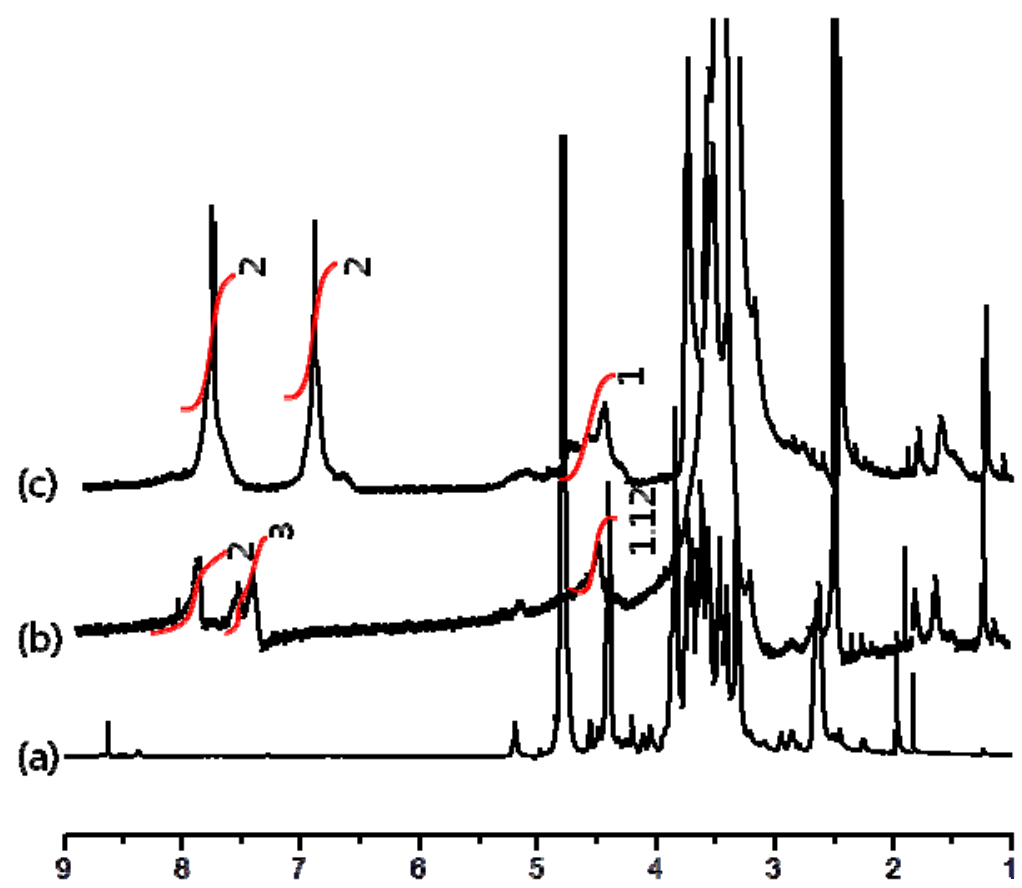

(a) CTS, (b) CTS-b, (c) CTS-m

The solubility of the products in organic solvents was tested using DMSO, DMF, acetone, ethanol, and THF. The results are shown in Table 2. Both of the synthesized benzoylated chitosan derivatives were soluble in DMSO, DMF, and acetone, but they were insoluble in THF and ethanol. 
Table 2. The solubility of the benzoyl chitosans in various organic solvents.

\begin{tabular}{cccccc}
\hline \multicolumn{7}{c}{ * Solubility of products } \\
\hline Solvent & DMSO & DMF & EtOH & Acetone & THF \\
CTS-b & $3.52 \mathrm{~g} / \mathrm{mL}$ & $3.44 \mathrm{~g} / \mathrm{mL}$ & IS & $1.54 \mathrm{~g} / \mathrm{mL}$ & IS \\
CTS-m & $3.30 \mathrm{~g} / \mathrm{mL}$ & $3.36 \mathrm{~g} / \mathrm{mL}$ & IS & $1.62 \mathrm{~g} / \mathrm{mL}$ & IS \\
\hline \multicolumn{7}{c}{ IS = insoluble. * Determined at r.t. }
\end{tabular}

Morphological studies were carried out using a SEM. As shown in Figure 2, the SEM images of the surface of the parent chitosan and the synthesized benzoyl chitosans show clear differences between them. The numerous pores in the parent chitosan are irregularly arranged and the diameter of the pores are roughly $0.5-2.0 \mu \mathrm{m}$. The shape of the pores is also comparatively uniform. However, the pores in the benzoylated chitosans are various in shape and are also much bigger in size $(5-20 \mu \mathrm{m})$. The magnified appearance of the parent chitosan looks like a piled fishing net, but that of benzoylated chitosans (benzoic acid and $p$-methoxybenzoyl chitosan) resembles slightly melted down coral-shaped dendritic structures. These images implies that the backbone of the chitosan benzoates-polymers undergoes significant structural changes after the acylation reaction, probably due to breaking of hydrogen bonds present in the parent chitosan and interaction between the newly introduced hydrophobic phenyl groups.

Figure 2. The SEM images of the parent chitosan and the synthesized benzoyl chitosans. The upper images: 25,000 times magnified images; The lower images: 5,000 times magnified images; Left: CTS; middle: CTS-b; right: CTS-m.
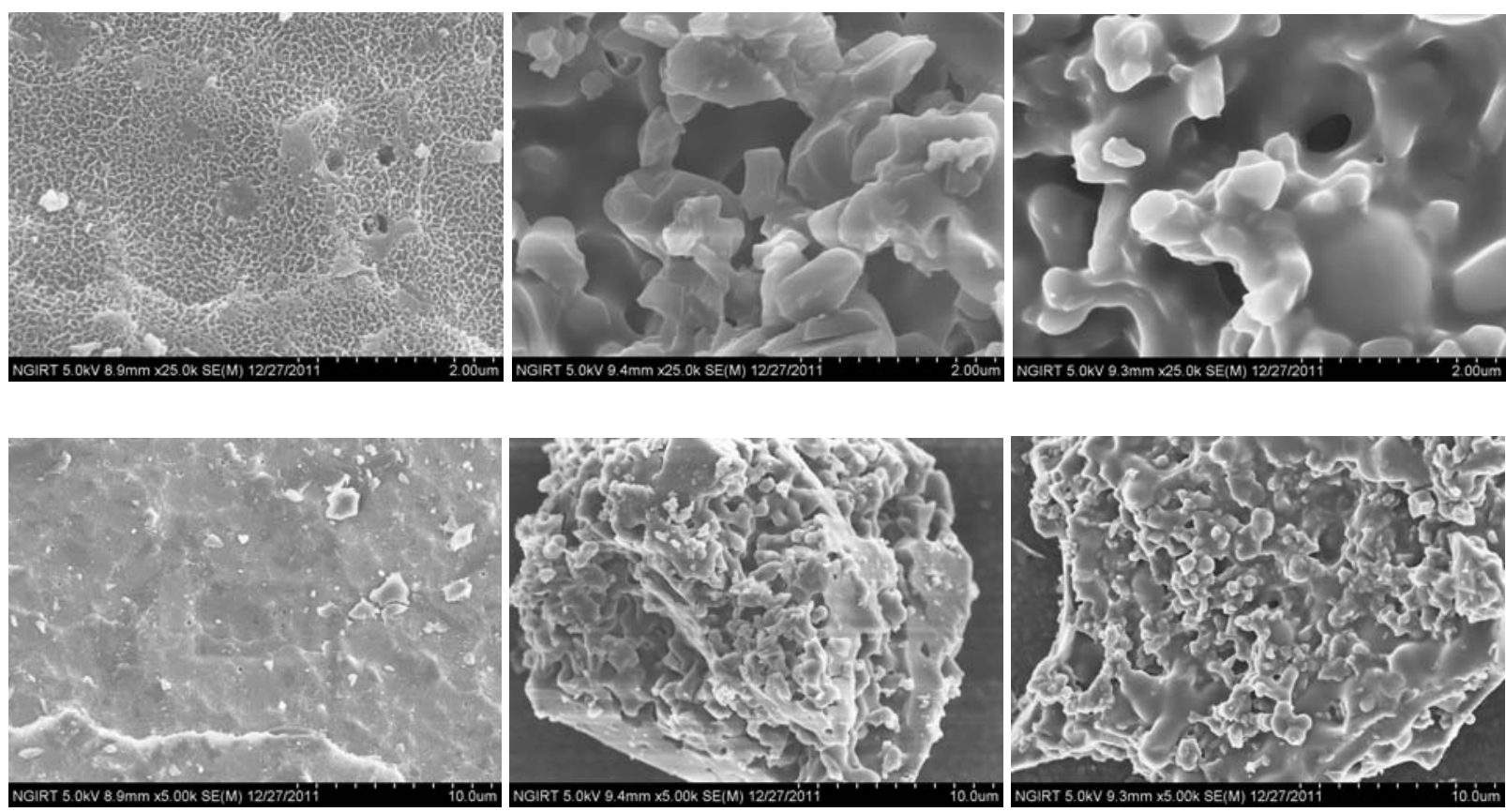


\section{Experimental}

\subsection{General}

The NMR spectra of the products were recorded at room temperature $\left(20^{\circ} \mathrm{C}\right)$ on a JEOL JNM-ECP FT-NMR spectrometer (500 MHz) using $5 \mathrm{~mm}$ diameter tubes. Samples were dissolved in DMSO- $d 6$ at the concentration of $15 \mathrm{mg} / \mathrm{mL}$. The IR spectra were recorded on a Shimadzu Prestige-21 FT-IR spectrometer. The samples were prepared as a $\mathrm{KBr}$ pallet and scanned against a blank $\mathrm{KBr}$ pellet background at wave number ranging from 4,000 to $400 \mathrm{~cm}^{-1}$. The surface morphology of the parent chitosan and the products were observed by scanning electron microscopy (SEM), using a S-4800 instrument (Hitachi, Tokyo, Japan). The samples were analyzed at an accelerating voltage of $5 \mathrm{kV}$ and $5,000-25,000 \times$ magnification. The solubility of the products was tested by dissolving a weighted sample in selected solvents at room temperature until the sample no longer became to be dissolved.

\subsection{Materials}

Chitosan (Mwt: below 1,000, degree of deacetylation: about 90\%, moisture content: 6.8\%) was purchased from the Kitto Lift (Pyeongtaek, Korea), TFAA (trifluoroacetic anhydride) from Acros Organics B.V.B.A (Geel, Antwerp, Belgium); benzoic acid and $p$-methoxybenzoic acid, 85\% phosphoric acid from Sigma Aldrich (Yongin, Korea); dimethyl sulfoxide- $d 6$ from Cambridge Isotope Laboratories, Inc. (Andover, MA, USA). All other chemicals are of reagent grade. The materials were used as received without further purification.

\subsection{Synthesis}

The chitosan benzoate was synthesized as follows: benzoic acid (1.44 g) was added portionwise to a flask containg TFAA $(6.56 \mathrm{~g})$ at $40{ }^{\circ} \mathrm{C}$ over $1 \mathrm{~h}$ with stirring. The reaction mixture was heated to $50{ }^{\circ} \mathrm{C}$ and stirred for further $1 \mathrm{~h}$ until the reaction mixture became a pale yellow transparent liquid. After the reaction temperature was lowered to $20{ }^{\circ} \mathrm{C}$ and $85 \%$ phosphoric acid $(0.343 \mathrm{~g})$ was added all at once to the solution and stirred for $10 \mathrm{~min}$. further, prior to addition of the chitosan $(1.0 \mathrm{~g}$, equivalent to $12.10 \mathrm{mmol}$ of hydroxyl groups). The reaction mixture was stirred at $20{ }^{\circ} \mathrm{C}$ for $0.5 \mathrm{~h}$ further, heated up to $45-50{ }^{\circ} \mathrm{C}$ and vigorously stirred for $20 \mathrm{~h}$ at the same temperature until it became a brown syrup. Anhydrous ethyl alcohol (about $5 \mathrm{~g}$ ) was added to the syrup and stirred for $10 \mathrm{~min}$. After volatile materials were completely removed by a rotary evaporator under reduced pressure, the solution was well mixed with anhydrous ethyl alcohol $(30 \mathrm{~mL})$ and filtered through a gauze cloth. The filtrate was again concentrated under reduced pressure to a syrup. The products were precipitated by dissolving the syrup in a $1: 1(\mathrm{v} / \mathrm{v})$ mixture of ethanol/acetone (total 10-15 $\mathrm{mL}$ ) followed by addition of diethyl ether $(300 \mathrm{~mL})$ and kept at a refrigerater $\left(0-5{ }^{\circ} \mathrm{C}\right)$ for $2-3 \mathrm{~h}$. The product was obtained as solid powder after filtration and washing several times with diethyl ether (18 L). The dissolving and washing processes were repeated more than 20 times until no colour appeared. The powder was dried at $50{ }^{\circ} \mathrm{C}$ under high vacuum for $5 \mathrm{~h}$. 
${ }^{1} \mathrm{H}-\mathrm{NMR}$ : for the chitosan $p$-methoxybenzoate (DMSO-d6 and 1 drop of $\mathrm{D}_{2} \mathrm{O}$ ): $\delta 7.9$ (doublet, aromatic Hs: ortho), $\delta 7.0$ (aromatic Hs: meta), $\delta 4.5$ (anomeric $\mathrm{C}-\mathrm{H}, 1.0 \mathrm{H}$ ), $\delta 3.2-3.9(\mathrm{C}-\mathrm{Hs}$ of carbohydrate backbone). Degree of substitution: 1.0 .

For the chitosan benzoate (DMSO- $d 6$ and 1 drop of $\mathrm{D}_{2} \mathrm{O}$ ): $\delta 7.95$ (broad singlet, aromatic Hs: meta), $\delta 7.6$ (broad singlet, aromatic Hs: para), $\delta 7.5$ (broad singlet, aromatic Hs: ortho), $\delta 4.5$ (anomeric C-H, 1.12 H), $\delta 3.2-3.9$ (C-Hs of carbohydrate backbone). Degree of substitution: 0.89.

FT-IR: For the chitosan benzoate (KBr pellet): around 3,450 $\mathrm{cm}^{-1}\left(\mathrm{~N}-\mathrm{H}\right.$ bonds), around 3,050 $\mathrm{cm}^{-1}$ (aromatic C-H bonds), 2,960 $\mathrm{cm}^{-1}$ (aliphatic $\mathrm{C}-\mathrm{H}$ bonds), 1,710 $\mathrm{cm}^{-1}$ (aromatic ester carbonyl), $1,270 \mathrm{~cm}^{-1}$ (aromatic C-H bonds).

For the chitosan $p$-methoxybenzoate $\left(\mathrm{KBr}\right.$ pellet): around $3,450 \mathrm{~cm}^{-1}(\mathrm{~N}-\mathrm{H}$ bonds), around $3,050 \mathrm{~cm}^{-1}$ (aromatic $\mathrm{C}-\mathrm{H}$ bonds), 2,960 $\mathrm{cm}^{-1}$ (aliphatic $\mathrm{C}-\mathrm{H}$ bonds), $1,710 \mathrm{~cm}^{-1}$ (aromatic ester carbonyl), 1,270 $\mathrm{cm}^{-1}$ (aromatic $\mathrm{C}-\mathrm{H}$ bonds).

\section{Conclusions}

A new synthetic method suitable for preparation of $O$-benzoyl chitosans, for not only academic small scale research, but also industrial mass production, has been developed using TFFA/ $\mathrm{H}_{3} \mathrm{PO}_{4}$-mediated acylation. The synthesized chitosan derivatives were soluble in organic solvents such as DMF, DMSO, Acetone, but they were all insoluble in THF and ethanol. The morphological study perfomed by SEM on the parent chitosan and the synthesized benzoyl chitosans suggests that the parent chitosan undergoes significant structural change after the benzoylation reaction. These structural changee in the benzoyl chitosans can be interpreted as a result of breaking of the hydrogen bonds and the hydrophobic interaction between newly introduced phenyl groups.

Thus, both of the synthetic method and the synthesized benzoyl chitosans, are expected to play an important role in the field of chitosan in future, especially for drug delivery, cosmetics, wound healing, and chromatographic separation technology, etc.

\section{Acknowledgements}

This work was financially supported by the Ministry of Education, Science Technology (MEST) and Korea Industrial Technology Foundation (KOTEF) through the Human Training Project for Regional Innovation.

\section{References and Notes}

1. Aranaz, I.; Mengíbar, M.; Harris, R.; Paños, I.; Miralles, B.; Acsosta, N.; Galed, G.; Heras, Á. Functional characterization of chitin and chitosan. Curr. Chem. Biol. 2009, 3, 203-230.

2. Rinaudo, M. Chitin and chitosan: Properties and applications. Prog. Polym. Sci. 2006, 31, 603-632.

3. Dutta, P.K.; Dutta, J.; Tripathi, V.S. Chitin and chitosan: Chemistry, properties and applications. J. Sci. Ind. Res. India 2004, 63, 20-31.

4. Kurita, K. Controlled functionalization of polysaccharide chitin. Prog. Polym. Sci. 2001, 26, 1921-1971. 
5. Majeti, N.V.; Kumar, R.A. Review of chitosan and chitosan applications. React. Funct. Polym. 2000, 46, 1-27.

6. Nishi, N.; Noguchi, J.; Tokura, S.; Shiota, H. Studies on chitin. 1. Acetylation of chitin. Polym. J. 1979, 11, 27-32.

7. Szosland, L. Di-O-butyrylchitin. In Chitin Handbook, Muzzareli, R.A.A., Peter, M.G., Eds.; European Chitin Society: Grottammare, Italy, 1997; pp. 53-60.

8. Yang, B.Y.; Ding, Q.; Montgomery, R. Preparation and physical properties of chitin fatty acids esters. Carbohydr. Res. 2009, 344, 336-342.

9. Batt, L.R.; Kim, B.M.; An, C.Y.; Lu, C.; Chung, Y.S.; Soung, M.G.; Park, S.H.; Chai, L.Y. Synthesis of chitin cycloalkyl ester derivatives and their physical properties. Carbohydr. Res. 2010, 345, 2102-2106.

10. Kaifu, K.; Nishi, N.; Komai, T. Preparation of hexanoyl, decanoyl, and dodecanoylchitin. J. Polym. Sci. Polym. Chem. Ed. 1981, 19, 2361-2363.

11. Sugimoto, M.; Kawahara, M.; Teramoto, Y.; Nishio, Y. Synthesis of acyl chitin derivatives and miscibility characterization of their blends with poly( $\varepsilon$-caprolactone). Carbohydr. Polym. 2010, 79, 948-954.

12. Somorin, O.; Nishi, N.; Tokura, S.; Nogichi, J. Studies on chitin. II. Preparation of benzyl and benzoylchitins. Polym. J. 1979, 11, 391-396.

13. Teramato, Y.; Miyata, T.; Nishio, Y. Dual mesomomorphic assemblage of chitin normal acylates and rapid enthalpy relaxation of their side chains. Biomacromolecules 2006, 7, 190-198.

14. Kankanala, K.; Reddy, V.R.; Mukkanti, K.; Pal, S. TFFA-H $\mathrm{PO}_{4}$-mediated direct, metal-free and high-speed synthesis of aryl carboxylate ester from phenols. J. Fluorine Chem. 2009, 130, 505-508.

15. Gray, A.D.; Smyth, T.P. Clean-chemistry synthesis of 2-tetralones in a single-stage acylation-cycloalkylation process. J. Org. Chem. 2001, 66, 7113-7117.

16. Smyth, T.P.; Corby, B.W. Toward a clean alternative to fridel-crafts acylation, in situ formation, observation, and reaction of an acyl bis(trifluoroacetyl)phosphate and related structures. J. Org. Chem. 1998, 63, 8946-8951.

17. Bhatt, L.R.; Kim, B.M.; Kim, H.; Kwak, G.B.; Lee, C.H.; Chai, K.Y. Preparation and characterization of chitin benzoic acid esters. Molecules 2011, 16, 3029-3036.

18. Bhatt, L.R.; Kim, B.M.; Kang, K.H.; Lu, C.; Chai, K.Y. Preparation of chitin butyrate by using phosphoryl mixed anhydride system. Carbohydr. Res. 2011, 346, 691-694.

19. Shibata, T.; Namikoshi, H.; Okamoto, I. Separation Agent Comprising Aliphatic or Aromatic Ester of Polysaccharide. U.S. Patent 5,268,098, 1993.

20. Okamoto, Y.; Hatada, K.; Shibata, T.; Namikoshi, H.; Okamoto, I. Optical Resolution with Tribenzoyl-B-1,4-chitosan. U.S. Patent 4,931,184, 1990.

21. Okamota, Y.; Yamamoto, C.; Kamigaito, M. Chitosan Derivative and Method of Producing Same. U.S. Patent 2009/0062519 A1, 2009.

22. Feng, Y.; Xia, W.; Shen, L.; Zhao, Y. Synthesis of $O$-benzoyl-chitosan and its antifungal activity. Shipin Yu Shengwu Jishu Xuebao 2011, 30, 367-370.

23. Vasnev, V.A.; Tarasov, A.I.; Markova, G.D.; Vinogradova, S.V.; Garkusha, O.G. Synthesis and properties of acylated chitin and chitosan derivatives. Carbohydr. Polym. 2006, 64, 184-189. 
24. Zhu, A.; Zhang, J.; Shen, J. Preparation and anticoagulant property of phosphorylcholineterminated $O$-benzoylchitosan derivative. J. Appl. Polym. Sci. 2003, 88, 489-493.

25. Wang, J.; Wang, H. Preparation of soluble $p$-aminobenzoyl chitosan ester by Schiff's base and antibacterial activity of the derivatives. Int. J. Biol. Macromol. 2011, 48, 523-529.

26. Hu, Y.; Du, Y.; Yang, J.; Tang, Y.; Li, J.; Wang, X. Self-aggreation and antibacterial activity of $N$-acylated chitosan. Polymer 2007, 48, 3098-3106.

27. Choi, C.Y.; Kim, S.B.; Park, P.K.; Yoo, D.I.; Chung, Y.S. Effect of $N$-acylation on structure and properties of chitosan fibers. Carbohydr. Polym. 2007, 68, 122-127.

28. Hirano, S.; Moriyasu, T. N-(Carboxyacy)chitosans. Carbohydr. Res. 1976, 47, 315-320.

29. Lee, K.Y.; Ha, W.S.; Park, W.H. Blood compatibility and biodegradability of partially $N$-acylated chitosan derivatives. Biomaterials 1995, 16, 1211-1216.

30. Hirano, S.O.; Ohe, Y.; Ono, H. Selective N-acylation of chitosan. Carbohydr. Res. 1976, 47, 315-320.

31. Kim, B.M.; Bhatt, L.R.; An, C.Y.; Kwak, G.B.; Lee, Y.H.; Kwon, T.-O.; Chai, K.Y. Synthesis and physical properties of chitosan long chain alkylester derivatives. J. Chitin Chitosan 2010, 15, 190-194.

Sample Availability: Samples of the compounds chitosan benzoates are available from the authors.

(C) 2012 by the authors; licensee MDPI, Basel, Switzerland. This article is an open access article distributed under the terms and conditions of the Creative Commons Attribution license (http://creativecommons.org/licenses/by/3.0/). 